\title{
An Evolutionary Approach to Camera-Based Projector Calibration
}

\author{
Clayton Matthew Johnson \\ Dept. of Math/Computer Science \\ California State University East Bay \\ Hayward, CA 94542 \\ matt.johnson@csueastbay.edu
}

\author{
Anu Bhat \\ Dept. of Math/Computer Science \\ California State University East Bay \\ Hayward, CA 94542 \\ anu_p_bhat@yahoo.com
}

\author{
William C. Thibault \\ Dept. of Math/Computer Science \\ California State University East Bay \\ Hayward, CA 94542 \\ william.thibault@csueastbay.edu
}

\begin{abstract}
In this paper, we describe a GA to solve for the orientation and lens characteristics of a projector, given images of a dome screen illuminated by the projector. We use synthetic images, making ground truth available. Our GA achieves rapid and accurate convergence.
\end{abstract}

\section{Categories and Subject Descriptors}

I.2.8 [Artificial Intelligence]: Problem Solving, Control Methods, and Search - Heuristic Methods; I.4.0 [Image Processing and Computer Vision]: General - Image Displays, Image Processing Software.

\section{General Terms}

Algorithms, Design.

\section{Keywords}

Genetic Algorithms, Projector-Camera Systems, Projector Calibration.

\section{INTRODUCTION}

Projectors are unique display devices that are able to place images of possibly very large size on almost any surface. When a precisely positioned image is needed, one often needs to know the precise location, orientation, and lens characteristics of the projector. Acquiring this information is called projector calibration. Recent approaches to projector calibration [1] use one or more digital cameras. The camera captures projections of known images, and correspondences are found between camera pixels and projector pixels. If the camera is calibrated, and the display surface geometry is known, then the projector calibration parameters can be determined. Once the projector is calibrated, it is possible to display objects at any desired display surface location. This is needed to support virtual reality applications where the viewer's head position is tracked, and an image correct for the users point-of-view is to be projected on the display surface.

Most work in projector calibration has dealt with flat display surfaces. This is a common situation, and allows application of techniques from the computer vision and photogrammetry literature. A combination of linear algebraic and non-linear optimization methods are typically used to calibrate projectors in the case of linear display surfaces[3,4,5]. Raskar has introduced a technique based on the quadratic eigenvalue problem [6] which

Copyright is held by the author/owner(s).

GECCO'06, July 8-12, 2006, Seattle, Washington, USA.

ACM 1-59593-186-4/06/0007. can be used to calibrate a projector using a spherical display surface.

Our work uses a GA to calibrate a projector using a spherical display surface and a calibrated camera. The projector is described by its intrinsic parameters (focal length, image center) and its extrinsic parameters (3D position and orientation). These parameters are expressed as matrices that describe how the projector maps image pixels to light rays. The mathematics of projection for projectors and cameras are identical. We use the pinhole camera model for the projector.

Points are represented in homogeneous coordinates. A 3D world point is related to the projector image point by the projection matrix $\mathbf{M}=\mathbf{K R}$, where $\mathbf{R}$ is the $3 \times 3$ rotation matrix determined by the three Euler angles defining the projector's orientation. We restrict the projector location to the origin, thus $\mathrm{R}$ describes the projector's extrinsic parameters. The intrinsic parameters are: $f_{1}$ and $f_{2}$, the projector's focal lengths; $\left(o_{x}, o_{y}\right)$ its principal point. These are arranged into a matrix $\mathbf{K}=\left[\begin{array}{ccc}f_{1} & 0 & o_{x} \\ 0 & f_{2} & o_{y} \\ 0 & 0 & 1\end{array}\right]$.

\section{EXPERIMENT}

We propose a unique evolutionary technique for performing camera-based projector calibration. We use a simple genetic algorithm as a global optimization technique to evolve a set of intrinsic and extrinsic parameters for the projector. The GA operates by minimizing the average displacement between the positions of the ideal and actual mappings of pixels. Our technique utilizes a software simulator to evaluate the calibration of the projector, thus providing an additional advantage over earlier approaches requiring physical equipment.

The experiment involved an uncalibrated single projector display system and a dome-shaped surface. The actual display system is represented as a projection matrix using the perspective projection model described above.

Each chromosome represents the following parameters of one possible projection model: $\theta, \Phi$ and $\psi$, the angular rotations (Euler angles) of the projector with respect to the projector coordinate system; $f_{1}$ and $f_{2}$, the components of the focal length of the lens; $o_{x}$ and $o_{y}$, the $\mathrm{x}$ and $\mathrm{y}$-coordinates of the principal point.

The COP is fixed at the origin, and is not encoded into the chromosome. 
Each member of the initial population is constructed by calling a random floating point number generator for each element in the chromosomes. As per IEEE specifications, a floating point variable is converted to a 32-bit binary string in its internal machine representation. The first bit of the float specifies its sign, the next sequence of eight expresses the exponent, and the remaining 23 bits represent the mantissa. We captured and used this internal representation as the genotypic representation of each parameter. Therefore, each binary chromosome is 224 bits long ( 7 parameters $\times 32$ bits) for the first experiment. The GA generated subsequent generations via tournament selection with elitism.

As part of this project, we developed a software simulator for use by the fitness function. The simulator makes is possible to compare the location on the dome screen of a pixel projected using the parameters in the chromosome, to the location that the same pixel is projected by the projector for which we have image data.

The projector is simulated by creating an intrinsic parameter matrix $\mathbf{K}$ and extrinsic matrix $\mathbf{R}$ from the seven elements contained within its chromosome. $\mathbf{M}$ is then calculated as the product of $\mathbf{K}$ and $\mathbf{R}$.

This projection is evaluated using a random sampling of 200 points in the projector image. For each of these points, the Euclidian distance between the ideal and actual coordinates is determined. The fitness of a chromosome is then calculated as the total of the distances between all 200 points. Thus, highly fit members of the population show the least amount of displacement and hence possess the lowest fitness values.

\section{RESULTS}

Several runs of the genetic algorithm were conducted using various crossover and mutation rates. Single, double-point and uniform crossover were considered. In most cases, the GA converged on a near optimal solution within fifty generations. The best results, described below, were evolved with a bitwise mutation rate of 0.0001 and uniform crossover.

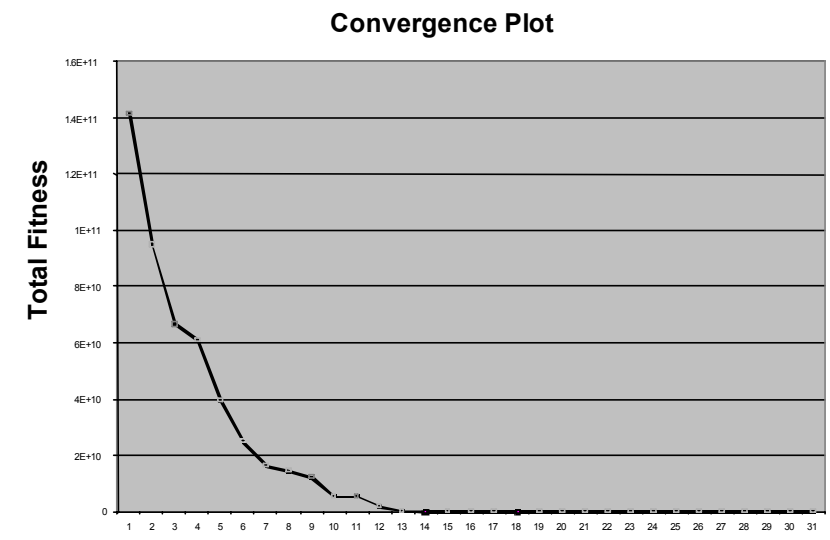

Generations

Figure 1. Convergence for projector calibration.

The convergence of the population is graphically depicted in Figure 1.
The total displacement between the ideal and actual mapping of pixels for the entire first generation was found to be $1.27 \cdot 10^{7}$.

At convergence, the final generation of the GA possessed a total pixel displacement of 5.080596. The best projection model showed a total error over 200 sampled points of only 0.00070761 .

\section{CONCLUSIONS}

Empirical results show that the proposed GA solution to camerabased projector calibration is quite effective at determining projection parameters. A very near optimal calibration of projectors was achieved within fifty generations of the algorithm.

The fitness function incorporated the use of a software simulation of a projector-camera system. This feature eliminated the need for conducting projector calibration trials upon the actual display surface. Furthermore, our approach is accurate even when the angle between the projector's principal axis and the display surface deviates widely from $90^{\circ}$.

We have shown that our approach works well on the relatively complex surface geometry of a dome structure.

\section{REFERENCES}

[1] Brown, M., Majumder, A., Yang, R., Camera-Based Calibration Techniques for Seamless Multiprojector Displays. In IEEE Transactions on Visualization and Computer Graphics, 11, 2, (March/April 2005).

[2] Chen, H., Sukthankar, R., Wallace, G., and Cham, T.J. Calibrating scalable multi-projector displays using camera homography trees. In Proceedings of the conference on visualization (VIS '02) (Boston, MA, Oct. 27 - Nov. 1, 2002). IEEE Computer Society Press, Washington, DC, 2002, 339-346.

[3] Chen, Y., Clark, D.W., Finkelstein, A., Housel, T.C., and Li, $\mathrm{K}$. Automatic alignment of high-resolution multi-projector displays using an un-calibrated camera. In Proceedings of the conference on visualization (VIS '00) (Salt Lake City, UT, Oct. 8-13, 2000). IEEE Computer Society Press, Washington, DC, 2000, 125-130.

[4] Raskar, R., Brown, M.S., Yang, R., Chen, W.C., Welch G., Towles, H., Seals, B., and Fuchs, H. Multi-projector displays using camera based registration. In Proceedings of the conference on visualization (VIS '99) (San Francisco, CA, Oct. 24-29, 1999). IEEE Computer Society Press, Los Alamitos, CA, 1999, 161-168.

[5] Raij, A., Gill G., Majumder, A., Towles, H., and Fuchs, H., PixelFlex2: A Comprehensive, Automatic, Casually-Aligned Multi-Projector Display. In Proceedings of IEEE International Workshop on Projector-Camera Systems (October 2003).

[6] Raskar, R., vanBaar, J., and Willwacher,T. Quadric Transfer for Immersive Curved Display, In Proceedings of EUROGRAPHICS 2004. 